\title{
TRATAMIENTO DE ORTODONCIA FIJA A PACIENTE ADULTO CON SÍNDROME DE PIERRE ROBIN - CASO CLÍNICO
}

\section{TREATMENT OF FIXED ORTHODONTICS TO AN ADULT PATIENT WITH PIERRE ROBIN'S SYNDROME - CLINICAL CASE}

\author{
Ramos-Montiel Ronald. ${ }^{1 *}$ \\ ${ }^{1}$ Docente de la Universidad Católica de Cuenca.Ecuador.Ecuador. \\ *ronald_mtz@hotmail.com
}

\begin{abstract}
Resumen
OBJETIVO. El síndrome de Pierre Robin (SPR) constituye un desafío diagnóstico antenatal, el cual se transforma en un desafío terapéutico posterior al nacimiento. Esta afección se caracteriza por presentar la mandíbula más pequeña de lo normal, la lengua replegada en la garganta y dificultad para respirar, este síndrome también se asocia a otras patologías. El tratamiento depende de la edad del paciente, la mayoría de los casos se desenvuelven quirúrgicamente para mejorar el desarrollo integral de cada individuo, pero las secuelas dento-alveolares quedan hasta edades adultas. Este es el reporte de un caso clínico que pretende demostrar el manejo del problema antes mencionado con aparatología ortondóntica fija en paciente adulto.
\end{abstract}

Palabras clave: PRS: Síndrome de Pierre Robin, micrognatia, glosoptosis.

\begin{abstract}
OBJECTIVE. Pierre Robin Syndrome (SPR) diagnosis is a challenge, which becomes a challenge after birth. This condition is characterized by the smallest normal jaw, tongue retracted in the throat and difficulty of breathing, this syndrome is also associated with other diseases. Treatment depends on the patient is age, most cases surgically operate to improve the integral development of each individual, but the patient presents dentoalveolar consequences until adulthood. This is the report of a case that seeks to demonstrate the management of these problems with orthodontics fixed in adult patient.
\end{abstract}

Key words: PRS: Pierre Robin syndrome, micrognathia, glossoptosis.

\section{INTRODUCCIÓN}

Las primeras descripciones de los niños nacidos con el síndrome se presentaron, en 1891 por Lannelongue y Menard, en 2 pacientes con micrognatia, paladar hendido y retroglosoptosis y luego, en 1923 por Pierre Robin, lo describió como un síndrome completo. ${ }^{1}$ Se denomina Secuencia a todos o algunos de los efectos secundarios a una de las anormalidades presentes en el paciente, en el caso del SPR, la micrognatia es la anomalía primaria y tanto el paladar hendido como la obstrucción de vía aérea, son secundarias a ella. ${ }^{2}$ El SPR es una anomalía congénita del arco branquial, por lo que estos pacientes desarrollan problemas respiratorios y digestivos severos desde el nacimiento, asociados a mortalidad elevada, debiendo iniciar el tratamiento adecuado para proteger la vía aérea superior. ${ }^{2,3} \mathrm{Al}$ ser una anomalía del primer arco branquial, se relaciona con múltiples problemas oftalmológicos, además de otros cuadros que pueden comprometer el posterior desarrollo del niño. ${ }^{3,4}$ Como consecuencia de la micrognatia, la lengua tiende a desplazarse hacia atrás produciendo obstrucción respiratoria, misma que provoca hipoxemia, hipercapnia, edema pulmonar, dificultad de la alimentación, vómitos, desnutrición, neumonías por aspiración y ocasionalmente la muerte. ${ }^{3,5}$ La precocidad y efectividad del manejo de esta secuencia es determinante, ya que la incoordinación de los mecanismos de succión y deglución, además de una inadecuada ventilación respiratoria, comprometen el estado nutricional y la calidad de vida del paciente. ${ }^{6}$

Existe varios factores externos que presionan al feto, estos pueden interferir con el crecimiento de la mandíbula 
y contribuir con el "SPR". Ciertas condiciones neurológicas que causan disminución del movimiento de la quijada en el útero pueden también pueden restringir el crecimiento de la mandíbula. Algunos estudios demuestran que puede haber una base genética para el "SPR". Los pacientes tratados a edades tempranas tienden a mejorar sus condiciones oro faciales al llegar a la edad adulta, pero la falta de desarrollo de la mandíbula puede provocar mal posiciones dentales permanentes.

\section{CASO CLÍNICO}

\section{EXAMEN EXTRAORAL DE INICIO.}

Paciente adulto, de sexo masculino de 18 años de edad, con excelente estado de salud, procedente de Durán, GuayasEcuador. Que acude a consulta acompañado de su madrina porque no es feliz con su sonrisa. Al examen clínico extra oral se observa perfil convexo severo (fig. 1A), una falta de desarrollo mandibular, asimetría facial y ocular, con ausencia notoria del mentón (fig. 1B), cara alargada, tercio inferior aumentado, forzamiento al cierre labial. (fig. 1C)
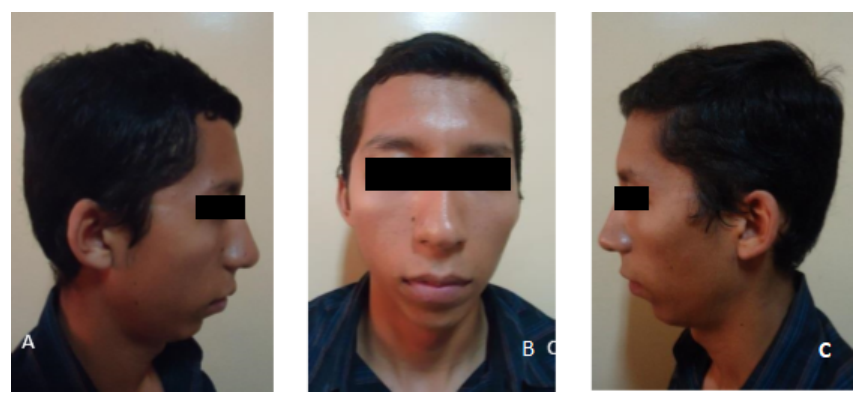

Fig. 1. Fotos extraorales: A. Perfil derecho B. Frontal. C Perfil izquierdo. Autoría: Ort. Esp. Ronald R. Ramos Montiel.

\section{EXAMEN INTRAORAL DE INICIO.}

Clínicamente se observa clase I canina derecha e izquierda, clase molar II derecha e izquierda (fig.2 A-C), ausencia de la pieza 1.1, una compresión maxilomandibular transversal, compensación dental al retrognatismo y enfermedad periodontal leve (fig. 2B). Se observa además lo característico del Biotipo Dolicofacial o leptoprosopo, las piezas dentales tienen forma alargada con unos vértices cuspídeos alargados que mejoran notoriamente los movimientos de lateralidad de la oclusión.
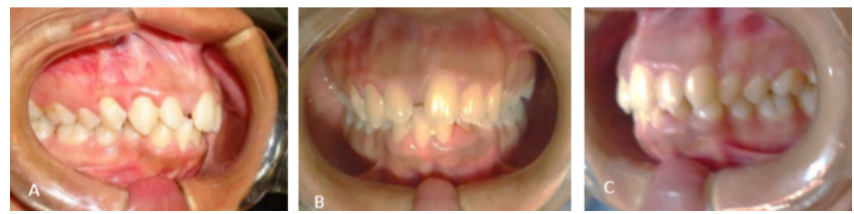

Fig. 2. Fotos intraorales: A. Derecho. B. Frente. C. Izquierdo. Autoría: Ort. Esp. Ronald R. Ramos Montiel.
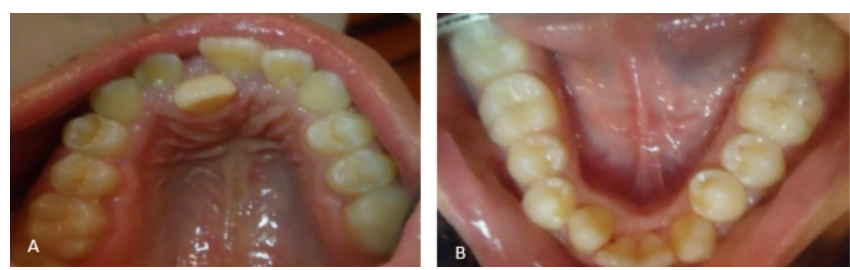

Fig. 3. Fotos intraorales: A. Arcada superior. B. Arcada inferior. Autoría: Ort. Esp. Ronald R. Ramos Montiel.

El maxilar superior presenta una arcada ovoidea y profunda, además se observa la palatalización de la pieza 1.1 colocándola en una situación completamente ectópica en relación al arco dentario, con apiñamiento severo (Fig. 3A). El maxilar inferior presenta una arcada triangular con ausencia de las piezas 3.2 y 4.2 apiñamiento moderado. (Fig.3B).

ESTUDIO RADIOGRÁFICO CEFALOMÉTRICO LATERAL.
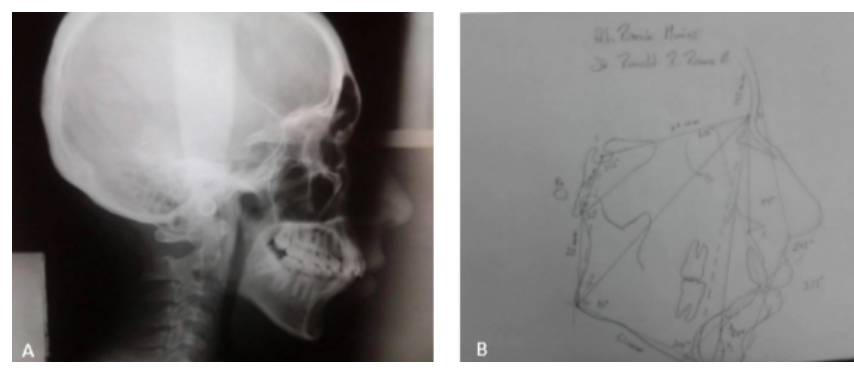

Fig. 4. A. Radiografía Cefalométrica lateral. B. Trazado de radiografía Cefalométrica lateral. Autoría: Ort. Esp. Ronald R. Ramos Montiel.

Se realizó un estudio Cefalométrico de Björk Jarabak, además de los ángulos de clase esqueletal del análisis de Steiner.

\begin{tabular}{|c|c|c|c} 
Angulo / Plano & NORMA & PACIENTE & DESCRIPCIÓN \\
\hline Silla & $123^{\circ}+/-5^{\circ}$ & $125^{\circ}$ & NORMA \\
\hline Artícular & $143+/-6^{\circ}$ & $165^{\circ}$ & RETROGNATISMO \\
\hline Goniaco Superior & $55^{\circ}+/-5^{\circ}$ & $36^{\circ}$ & RETROGNATISMO \\
\hline Goniaco Inferior & $75^{\circ}+/-5^{\circ}$ & $75^{\circ}$ & NORMA \\
\hline IS-SN & $104^{\circ}+/-3^{\circ}$ & $106^{\circ}$ & NORMA \\
\hline II/GO-GN & $90^{\circ}+/-4^{\circ}$ & $107^{\circ}$ & PROINCLINACIÓN \\
\hline SNA & $82^{\circ}+/-2^{\circ}$ & $74^{\circ}$ & \multirow{2}{*}{ CLASE ESQUELETAL II } \\
\hline SNB & $80^{\circ}+/-2^{\circ}$ & $64^{\circ}$ & \multirow{2}{*}{ MAYORES POSIBILIDADES } \\
ANB & $+2^{\circ}+/-2^{\circ}$ & $+10^{\circ}$ & DE EXTRACCIÓN \\
\hline IS/II & $131^{\circ}+/-7^{\circ}$ & $121^{\circ}$ & PERFIL CONVEXO \\
\hline Perfil Facial & $170^{\circ}-180^{\circ}$ & $148^{\circ}$ & NORMA \\
\hline BCP & $32 \mathrm{~mm}+/-3 \mathrm{~mm}$ & $33 \mathrm{~mm}$ & NORMA \\
\hline BCA & $71 \mathrm{~mm}+/-3 \mathrm{~mm}$ & $77 \mathrm{~mm}$ & CUERPO MANDIBULAR \\
\cline { 1 - 1 } LCM & $71 \mathrm{~mm}+/-3 \mathrm{~mm}$ & $63 \mathrm{~mm}$ & PEQUEÑO \\
\hline AFP & $70 \mathrm{~mm}$ & $85 \mathrm{~mm}$ & AUMINUIDO \\
\hline AFA & $105 \mathrm{~mm}-120 \mathrm{~mm}$ & $145 \mathrm{~mm}$ & DOLICOFACIAL
\end{tabular}

Tabla 1. Resultado de análisis Cefalométrico de Björk Jarabak, además de los ángulos de clase esqueletal del análisis de Steiner. 


\section{ESTUDIO RADIOGRÁFICO PANORÁMICO.}
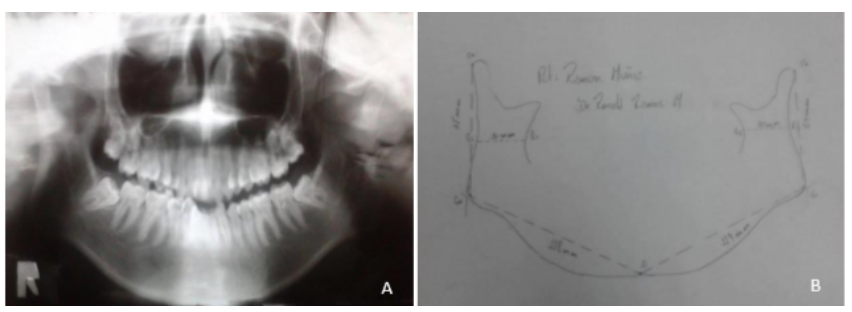

Fig. 5. A. Radiografía Panorámica. B. Trazado de radiografía panorámica. Autoría: Ort. Esp. Ronald R. Ramos Montiel.

\begin{tabular}{|l|l|l|l}
\hline PLANOS & DERECHO & IZQUIERDO & DIFERENCIAS \\
\hline CO-GO & $75 \mathrm{~mm}$ & $69 \mathrm{~mm}$ & $6 \mathrm{~mm}$ \\
\hline GO-SI & $118 \mathrm{~mm}$ & $119 \mathrm{~mm}$ & $1 \mathrm{~mm}$ \\
\hline R1-R2 & $30 \mathrm{~mm}$ & $30 \mathrm{~mm}$ & $0 \mathrm{~mm}$ \\
\hline
\end{tabular}

Tabla 2. Tabla2: Resultado de análisis de Radiografía Panorámica. Autoría: Ort. Esp. Ronald R. Ramos Montiel.

\section{DIAGNÓSTICO CEFALOMÉTRICO.}

1) Paciente presenta retrognatismo

2) Clase II Esqueletal

3) Proinclinación dento-alveolar inferior

4) Mayores posibilidades de extracciones

5) Perfil Convexo

6) Cuerpo Mandibular Pequeño

7) Biotipo Dolicofacial

8) Paciente asimétrico funcional

\section{DIAGNÓSTICO GENERAL DEL PACIENTE.}

Paciente adulto, de sexo masculino de 18 años de edad, Clase II Esqueletal por retrognatismo, cuerpo mandibular pequeño, perfil convexo, asimetría facial y ocular, con ausencia notoria del mentón, cara alargada, tercio inferior aumentado, forzamiento al cierre labial, palatalización de la pieza 1.1 colocándola en una situación completamente ectópica en relación al arco dentario superior, con apiñamiento severo. Proinclinación dentoalveolar inferior con una arcada triangular inferior y ausencia de las piezas 3.2 y 4.2 apiñamiento moderado. Paciente asimétrico funcional.

\section{PLAN DE TRATAMIENTO.}

1) Alinear y nivelar.

2) Crear espacio para recuperar pieza 1.1 Paralelizar raíces.

3) Contención removible superior e inferior

\section{ETAPAS DEL TRATAMIENTO.}

Cementado de la aparatología ortodóncica fija superior e inferior, empezando con arcos Nitinol livianos, y un resorte de Nitinol entre $2.1-1.2$ (Fig. 6A, B, C).
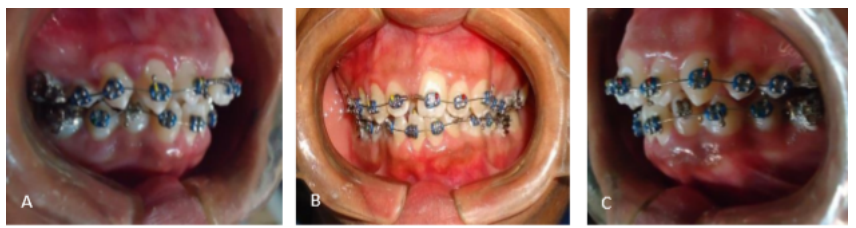

Fig. 6. Fotos intraorales de inicio de tratamiento: A. Derecho. B Frente. C Izquierdo. Autoría: Ort. Esp. Ronald R. Ramos Montiel.

A los 4 meses se consigue el espacio necesario para la recuperación de la pieza dental 1.1 al arco dentario (Fig. 7B); además, se colocan topes oclusales en los primeros molares inferiores para el levante de la mordida (Fig. 7 A, C, D).
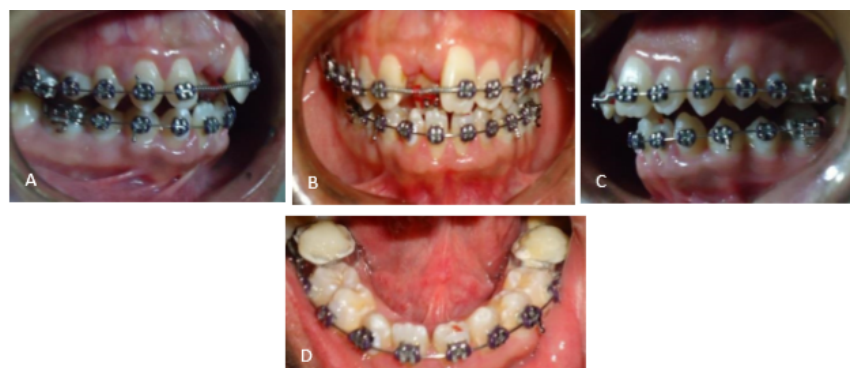

Fig. 7. Fotos intraorales durante el tratamiento: A. Derecho. B Frente. C Izquierdo. D Oclusal. Autoría: Ort. Esp. Ronald R. Ramos Montiel.

Luego de 12 meses de tratamiento se recuperó por completo el espacio para ubicar correctamente la pieza dental 1.1 manteniendo las clases caninas (con la ausencia de las $3.2,4.2)$ y molares; además de la eliminación de los topes oclusales; además de esto se adicionaron ligas Clase II para mejorar4 posición mandibular. (Fig. 14)
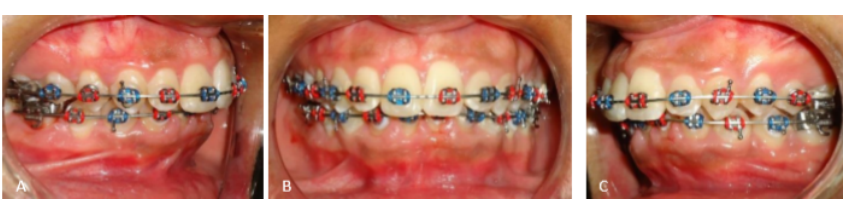

Fig. 8. Fotos intraorales durante el tratamiento: A. Derecho. B Frente. C Izquierdo. Autoría: Ort. Esp. Ronald R. Ramos Montiel.

El tratamiento tuvo una duración de 14 meses en lo cual incluyo un ajuste Oclusal y un alargamiento de corona de la piza dental 1.1, debido a que la recuperación de la misma, provoco una almohadilla gingival, no se colocó contención 
debido al completo equilibrio dental intermaxilar.

\section{EXAMEN EXTRAORAL FINAL.}

Se puede observar lo notables cambios como mayor tonicidad muscular labial, lo cual mejoro el cierre labial forzado (Fig. 9: A, B). Claramente se presenta el avance mandibular (Fig. 9: C, D; Fig. 10:E, F); además de ellos se adquirió una sonrisa armónica con su rostro. (Fig. 10: G, H).
ANTES
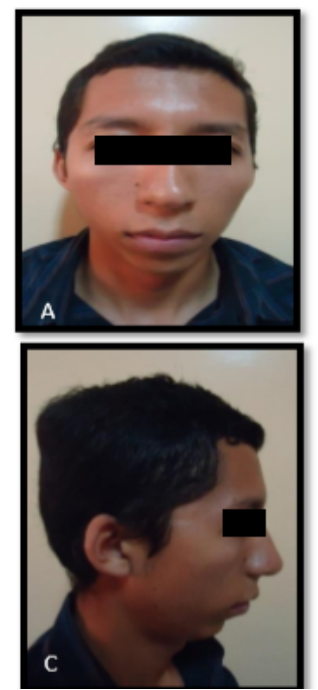

DESPUES
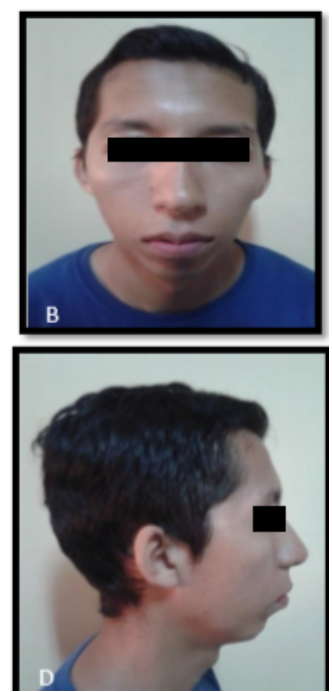

Fig. 9. Comparativo de paciente extraoral: A. Frente antes. B. Frente después. C. Perfil antes. D: Perfil después. Autoría: Ort. Esp. Ronald R. Ramos Montiel.
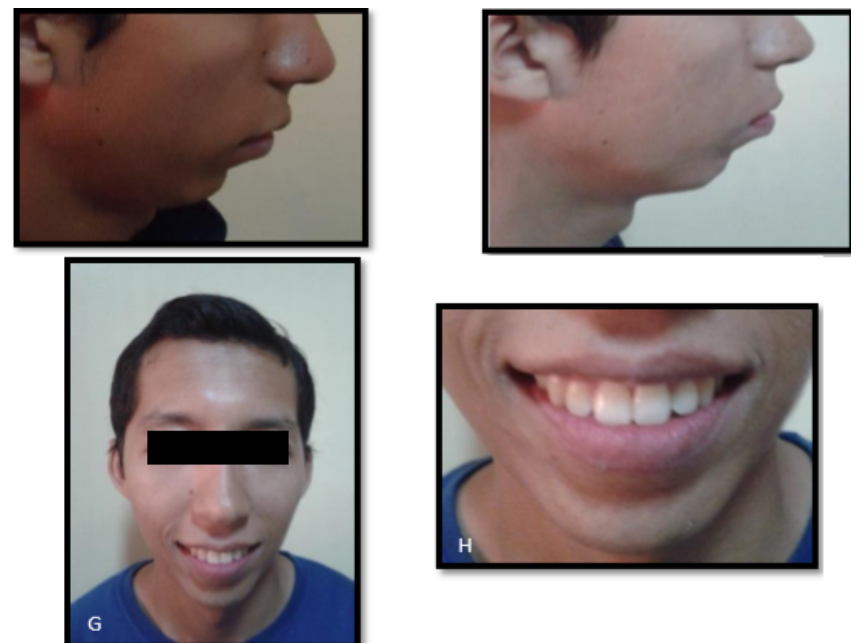

Fig. 10. E: Tercio inferior antes. F: Tercio inferior después. G: Rostro con sonrisa de paciente. H: Sonrisa de paciente. Autoría: Ort. Esp. Ronald R. Ramos Montiel.

\section{EXAMEN INTRAORAL FINAL.}

Se observa una equilibrada estética dental, además de una correcta coordinación inter-arcada dentaria superior e inferior, manteniéndose una clase canina y molar I, estableciéndose una oclusión mutuamente protegida. Las arcadas dentarias superior e inferior presentan una forma ovoidea, lo cual elimina la tendencia a recidiva dentaria.
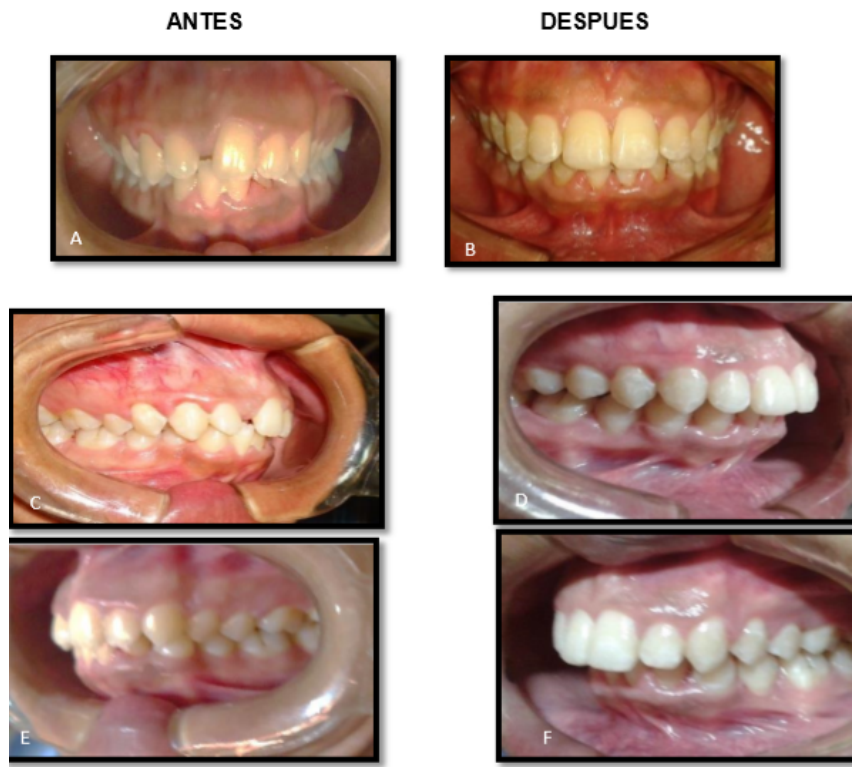

Fig. 11. Comparativo de paciente intraoral: A. Frontal antes. B. Frontal después. C. Derecho antes. D: Derecho después. E: Izquierdo antes. F: Izquierdo después. Autoría: Ort. Esp. Ronald R. Ramos Montiel.
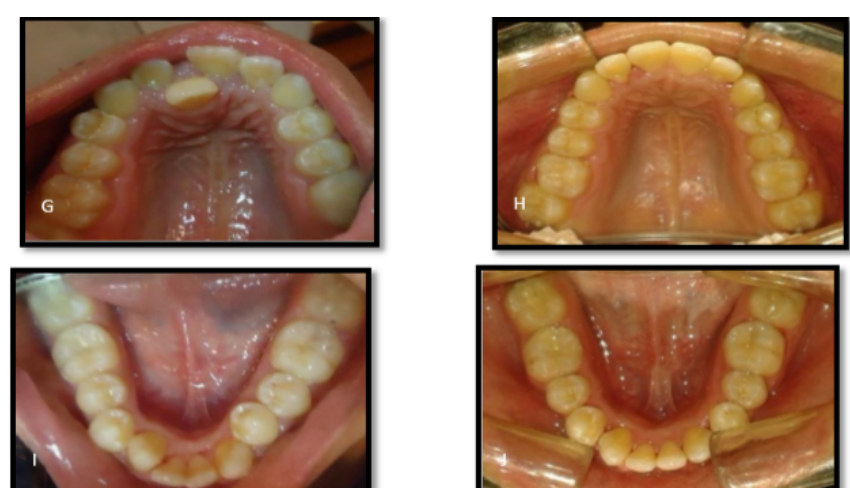

Fig. 12. G: Oclusal superior antes. H: Oclusal superior después. I: Oclusal inferior antes. H: Oclusal inferior después. Autoría: Ort. Esp. Ronald R. Ramos Montiel.

\section{RESULTADOS.}

1) La correcta alineación dento-alveolar modifica la posición de la lengua.

2) Elimina los problemas respiratorios. 
3) Se mejoró la deglución.

4) Desaparición de los fenómenos de reflujo.

\section{CONCLUSIÓN.}

1) Aunque el SPR debe ser trata do en la niñez; no se lo hace por falta de conocimiento, lo cual presenta secuelas en la edad adulta.

2) El cambio es eminentemente dentoalveolar.

3) Debido al cuidado en las fuerzas emitidas durante el tratamiento de ortodoncia fija el tratamiento finalizo en un tiempo de 14 meses.

4) No se colocó contención en el paciente debido a que con la oclusión mutuamente protegida y una musculatura equilibrada conseguida al final del tratamiento era poco probable la presencia de una recidiva.

5) La forma final de las arcadas ovoides permitieron una interrelación óptima en la oclusión del paciente permitiendo de esta manera un mayor número de puntos de contacto.

6) Existen un Bolton Anterior por la ausencia de las piezas $3.2-4.2$.

7) Podría tener implicancias periodontales posteriores con el pasar del tiempo.

\section{Referencias}

1 Morovi G. Manejo actual en síndrome de Pierre Robin. Rev Chil Pediatr 2004; 75 (1); 36-42.

2 Herrera GR. Manejo de la vía aérea en pacientes con secuencia de Pierre Robin. Revista Hospitales de Costa Rica. 1997;7:193-4.

3 Pérez-González JA, García-Cartaya Z. Síndrome de Pierre Robin. Presentación de un caso clínico. Panorama Cuba y Salud. 2011;6(1):44-6.

4 Agresti A, Caffo B. Simple and effective confi dence intervals for proportions and differences of proportions result from adding two successes and two failures. J of American Statistics Assoc. 2000;54(4):280-8.

5 Baujat G, Faure C, Zaouche A, Viarme F, Couly G, Abadie V. Oroesophageal motor disorders in Pierre Robin syndrome. J Pediatr Gastroenterol Nutr. 2001;32(3):297-302.

6 Marques IL, De Sousa TV, Careiro A, Peres S, Barbieri MA, Bettiol H. Sequiência de Robin: protocolo único de tratamento. Jornal de Pediatría. 2005;81(1):14-22.

Recibido: 16 de mayo de 2018.

Aceptado: 27 de mayo de 2018. 
